Document downloaded from:

http://hdl.handle.net/10251/109189

This paper must be cited as:

Quiñones, DR.; Cuevas-López, A.; Cambra-Enguix J.; Canals-Gamoneda, S.; Moratal, D. (2017). RATT: RFID Assisted Tracking Tile. Preliminary results. Proceedings Intenational Anual Conference of IEEE Engineering in Medicine and Biology Society. 4114-4117. doi:10.1109/EMBC.2017.8037761

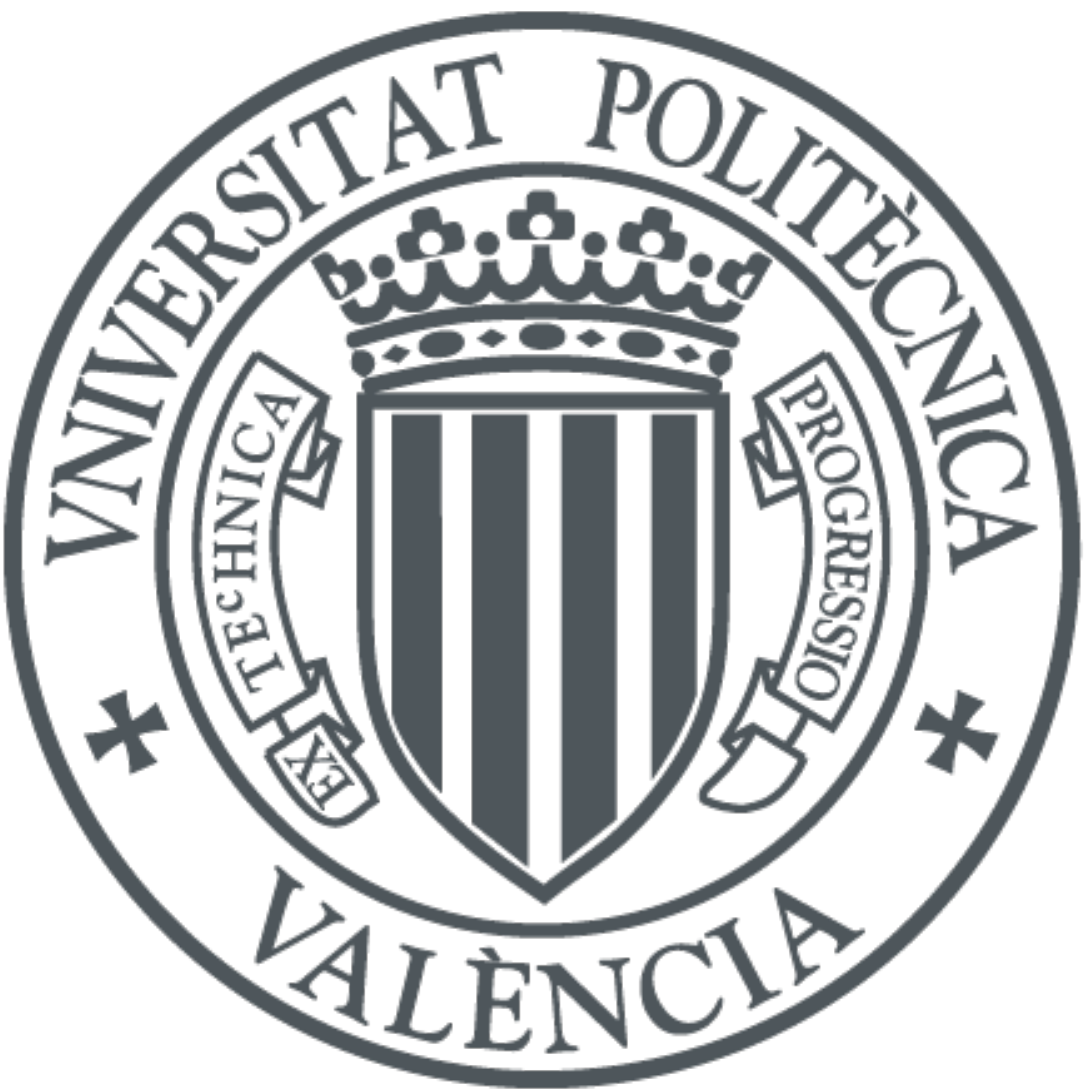

The final publication is available at

http://doi.org/10.1109/EMBC.2017.8037761

Copyright IEEE Engineering in Medicine and Biology Society

Additional Information 


\title{
RATT: RFID Assisted Tracking Tile. Preliminary Results
}

\author{
Darío R. Quiñones, Aarón Cuevas, Javier Cambra, \\ Santiago Canals, and David Moratal, Senior Member, IEEE
}

\begin{abstract}
Behavior is one of the most important aspects of animal life. This behavior depends on the link between animals, their nervous systems and their environment. In order to study the behavior of laboratory animals several tools are needed, but a tracking tool is essential to perform a thorough behavioral study. Currently, several visual tracking tools are available. However, they have some drawbacks. For instance, when an animal is inside a cave, or is close to other animals, the tracking cameras cannot always detect the location or movement of this animal.
\end{abstract}

This paper presents RFID Assisted Tracking Tile (RATT), a tracking system based on passive Radio Frequency Identification (RFID) technology in high frequency band according to ISO/IEC 15693.

The RATT system is composed of electronic tiles that have nine active RFID antennas attached; in addition, it contains several overlapping passive coils to improve the magnetic field characteristics. Using several tiles, a large surface can be built on which the animals can move, allowing identification and tracking of their movements. This system, that could also be combined with a visual tracking system, paves the way for complete behavioral studies.

\section{INTRODUCTION}

Experimentation in animals, or in vivo testing, involves the use of model organisms as substitutes for humans. These types of tests are frequently used to investigate the causes and treatments of human diseases [1-2]. However, carrying out these experiments in humans is contrary to bioethics. For this reason, a model organism is used to study specific biological phenomena that can be extrapolated to other species [3-4].

In the case of research for neuroscience, the most frequently used model organisms are rats and mice. This is because of their high rate of reproduction, their ease of handling and the great number of physiological similarities that they share with human beings. Therefore, the experimental conditions have to be adapted to the size and characteristics of these animals [5].

* Research supported in part by the Spanish Ministerio de Economía y Competitividad (MINECO) and FEDER funds under grants BFU201564380-C2-2-R and BFU2015-64380-C2-1-R. Santiago Canals acknowledges financial support from the Spanish State Research Agency, through the "Severo Ochoa" Programme for Centres of Excellence in R\&D (ref. SEV- 2013-0317). Darío R. Quiñones is supported by grant Ayudas para la formación de personal investigador (FPI) from Universitat Politècnica de València.

D.R. Quiñones, A. Cuevas and J. Cambra are with the Center for Biomaterials and Tissue Engineering, Universitat Politècnica de València, 46022 Valencia, Spain.

S. Canals is with the Neuroscience Institute, Consejo Superior de Investigaciones Científicas - CSIC, San Juan de Alicante, Spain.

D. Moratal is with the Center for Biomaterials and Tissue Engineering, Universitat Politècnica de València, 46022 Valencia, Spain (phone: 96.387.70.00 ext. 88939; e-mail: dmoratal@eln.upv.es).
Huge numbers of studies rely on animals moving freely within a controlled environment [5-7]; for instance, those related to behavioral or memory research [6-7]. The configuration of the environment may vary according to the needs of the experiment [10-11]. In addition, they may have devices that react to the presence or actions of the subjects.

Knowing the positions of different animals within the space is essential. However, it is difficult for current visual tracking systems to locate the animals when they are in groups or inside a burrow.

While current visual tracking systems are useful, having an automatic tracking system [13], which facilitates data collection by filtering events of interest to the researcher, would provide greater accuracy.

The RFID Assisted Tracking Tile (RATT) system we present in this paper allows tracking with a high number of subjects, enabling easy identification of each one at each moment. Moreover, it overcomes the drawback of losing the localization of the animals when they are in places where the camera cannot satisfactorily record. Finally, combining current visual tracking systems with the RATT system will improve the quality of the results of behavior research studies.

\section{MATERIALS AND METHODS}

\section{A. RATT Concept}

In order to investigate animals in controlled conditions, an automated experimentation environment was created as shown in Fig. 1. This modular environment recreates the natural conditions of the animals to collect data from that are impossible to observe in current closed environments. One RATT environment is formed using several RATT tiles making the device easy to replicate and extend.

Each RATT tile (Fig. 2) consists on one main Control

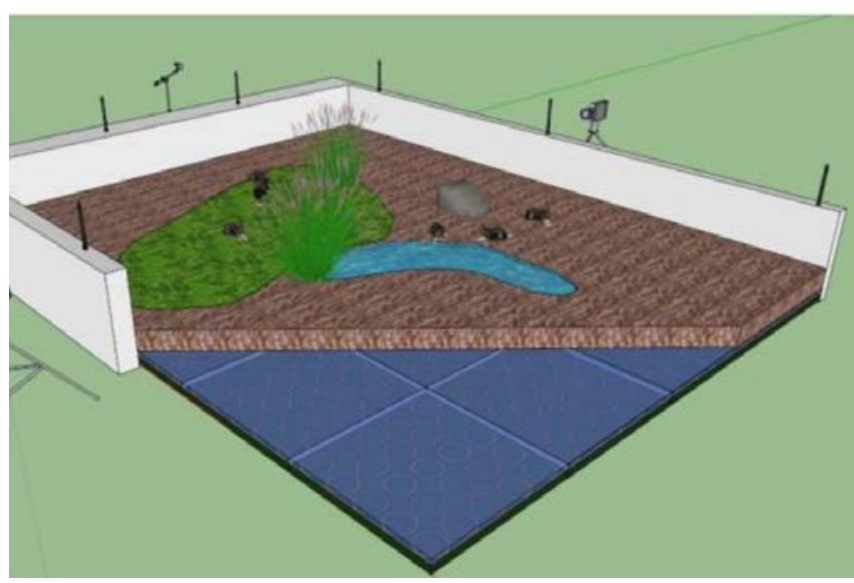

Figure 1. Virtual animal environment 


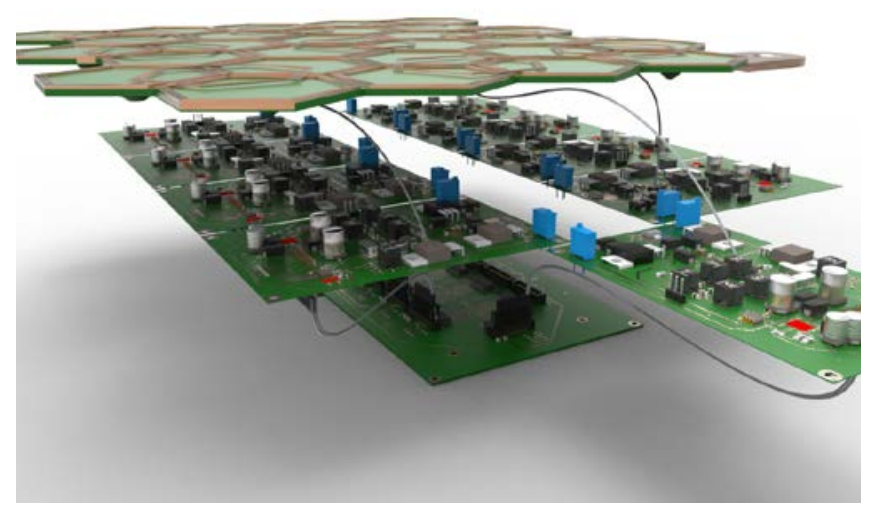

Figure 2. Three-dimensional design of one RATT tile

board and nine RF Boards as shown in Fig. 3. The Control board is the core of whole system and it has the function of controlling the nine Power amplifier boards and providing the UDP communication protocol.

\section{B. LPC ARM}

To control the entire system an LPC4088 from Embeded artists (Davidshallsgatan, Malmö, Sweden) was used. It has an ARM Cortex-M4F at a frequency of $120 \mathrm{MHz}$, with $96 \mathrm{~KB}$ of RAM and $512 \mathrm{~KB}$ of FLASH integrated into the chip. It also has SPI, UART or I2C buses. This board contains USB and Ethernet ports, providing the necessary entire physical layer. The power is limited to $5-5.5 \mathrm{~V}$ and the output regulator is shared, allowing external devices to be connected.

\section{Control Board}

A main board to allocate the LPC4088, a 5V power supply, connectors and communication buses was designed (Fig. 4). The 5V power supply provides 3.5A for the electronic components. An auxiliary I2C bus was integrated into the PCB.

Communication with the nine RF boards is made through a parallel data bus with chip select control lines, so the RFID transceivers on each board can be controlled independently. Additionally, an I2C bus allows control of auxiliary features, like digital output power control.

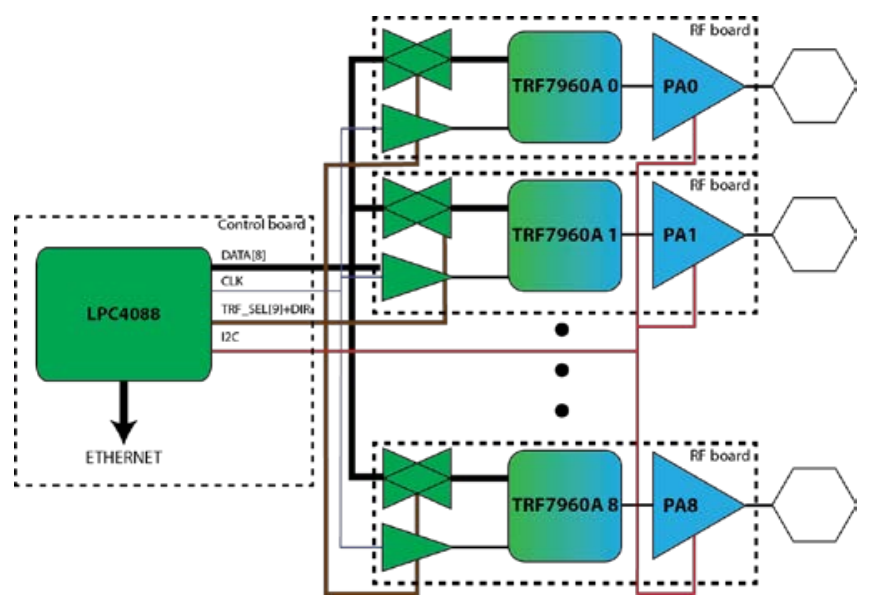

Figure 3. System overview

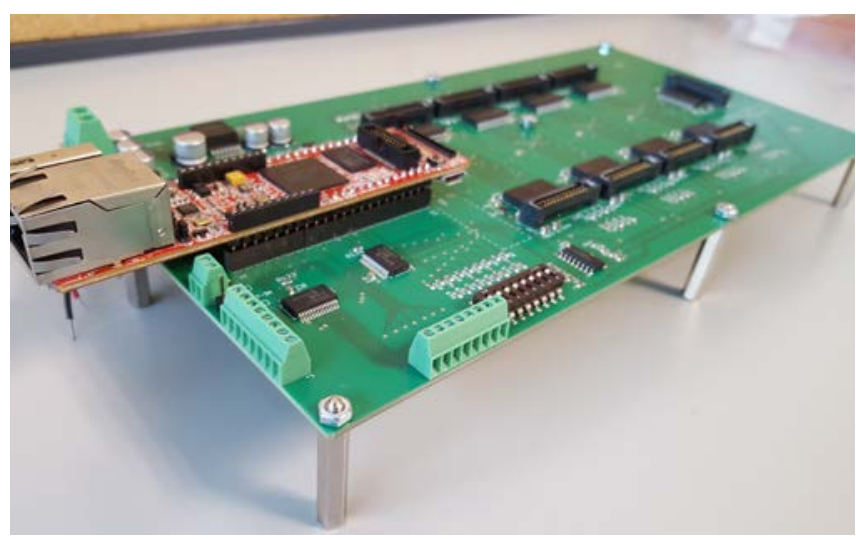

Figure 4. Control Board

To attach nine RF Boards to the Control Board nine connectors with only one plug position were used. This ensures that the $R F$ Boards are connected properly. Moreover, to prevent malfunction, a power indicator line was created. This power indicator line turns off all the systems in case the external power supply is disconnected.

\section{RF Board}

The RF board (Fig. 5) features a digitally controlled RFID transceiver and a radiofrequency power amplifier. The used transceiver is a TRF7960A (Multi-Protocol Fully Integrated 13.56-MHz RFID Reader/Writer) transceiver from Texas Instruments (Dallas, Texas, USA). The power amplifier is based on that featured in an application note from the transceiver manufacturer. It is a class E amplifier connected with impedance-matching circuits to a $50 \Omega$ antenna.

This amplifier has an emission power of up to $4 \mathrm{~W}$. The output power is set by a digital potentiometer, which is controlled by software. The device is controlled via the I2C bus and is connected to the global reset line so that it selects a minimum power for the amplifier as a safety measure in case the digital supply of the tile is not correct.

\section{E. RFID Tags}

RF-HDT-DVBB tags from Texas Instruments were used for development and testing. These transponders are compatible with the ISO/IEC 15693 standard requiring a field of $112 \mathrm{~dB} \mu \mathrm{A} / \mathrm{m}$ for activation and encapsulated in the form of a $3 \mathrm{~cm}$ diameter coin. Therefore, they are unable to be located inside the animals' bodies. Nevertheless, this problem was solved with standard units enclosed in biocompatible glass as shown in Fig. 6.

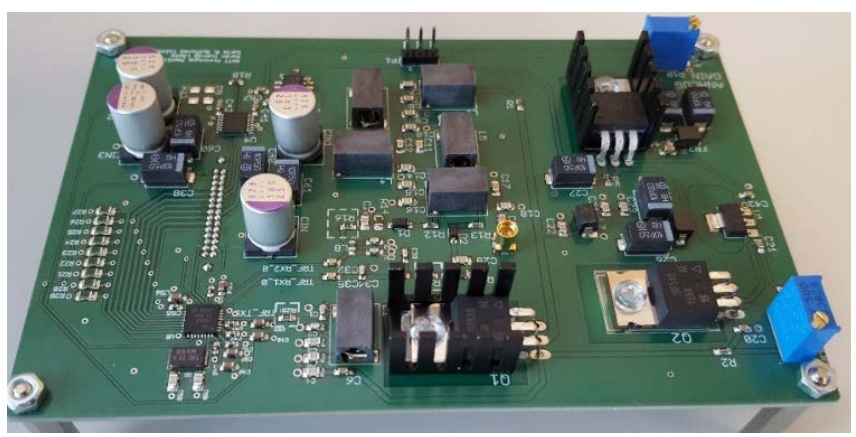

Figure 5. RF Board 


$\begin{array}{lll}\text { I-CODE SLI } & \begin{array}{l}\text { I-CODE SLI } \\ 3 \times 13 \mathrm{~mm}\end{array} & \begin{array}{l}\text { I-CODE SLI } \\ 4 \times 22 \mathrm{~mm}\end{array} \\ & & \end{array}$

Figure 6. Cristal RFID tags

\section{F. UDP communication}

Each electronic tile uses the Ethernet (Fig. 7) connection to connect to a control computer; it receives the information from the different tiles to perform the necessary triangulation calculations and provides control and synchronization functions.

\section{G. RFID Antennas}

Hexagonal shaped antennas were developed for the RFID tags detection as shown in Fig. 8. This shape was chosen to optimize the space and the magnetic field distribution. Three antennas were positioned in three different rows at the bottom of the tile. A grid of passive tuned coils were positioned on top of each tile in order to improve the magnetic field characteristics.

\section{H. Triangulation}

The triangulation software displays a map of the tile and the position of each detected tag. The image is refreshed periodically, each time a new set of positional data is received. To calculate each tag coordinates, the diffusion algorithm [14] is used.

This algorithm performs a weighted sum for each detected tag, as shown in Equation 1. Pi and wi represent the center positions of the $i$-th antenna and a function of the tag signal strength it detects.

$$
(x, y)=\frac{\sum_{i=1}^{N} w_{i} P_{i}(x, y)}{\sum_{i=1}^{N} w_{i}}
$$

\section{REsults AND DiscussiOn}

Simultaneous localization of multiple RFID tags was achieved. In this way it was possible to obtain readings as shown in Fig. 9, which shows the relationship between the various real positions of the tags and those measured by the system.

An interesting result is the existence of inductive interaction between tags when they are close together. These create variations in RSSI (received signal strength indication)

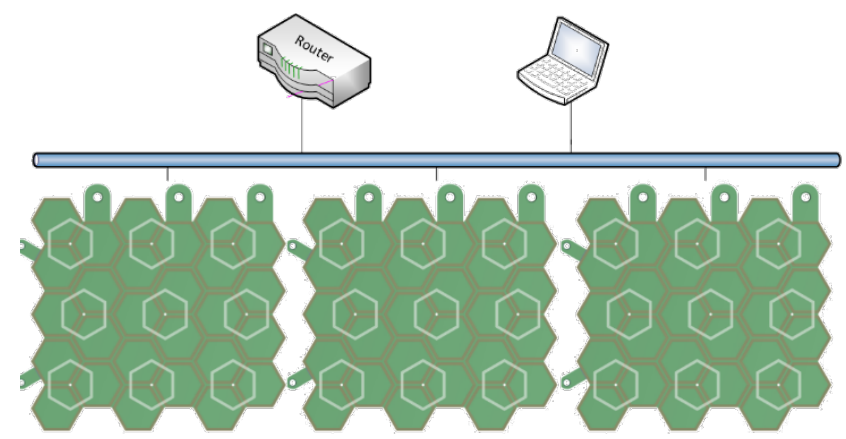

Figure 7. Diagram of connection between multiple tiles and an Ethernet network for the transmission of data to the computer.

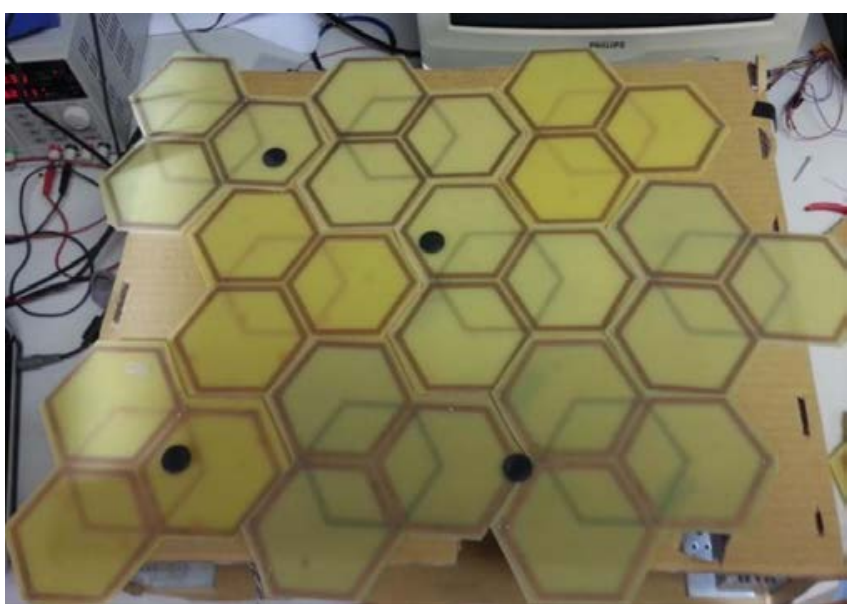

Figure 8. Complete RATT tile.

readings. In this way, and with the transceivers used, three zones can be identified:

- $\quad$ Tags distant from each other $(>10 \mathrm{~cm})$ : This case offers the best resolution, with a maximum positioning error of $3 \mathrm{~cm}$.

- $\quad$ Medium distance tags $(5-10 \mathrm{~cm})$ : In this case, the positioning error increases up to $5 \mathrm{~cm}$, maintaining the relative positions of the tags.

- $\quad$ Short distance tags $(<5 \mathrm{~cm})$ : When the tags are so close, the error is still $5 \mathrm{~cm}$ but the relative positions are not maintained.

These tests were performed with the $3 \mathrm{~cm}$ RFID coin
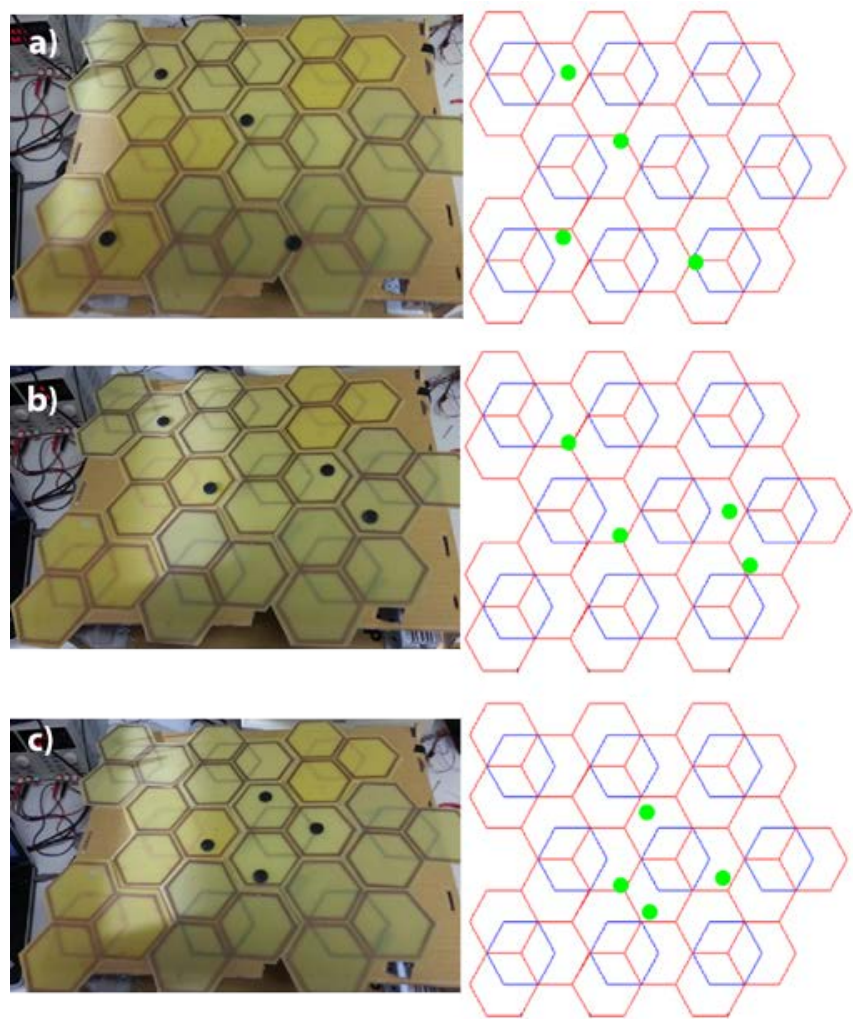

Figure 9. RATT testing with 4 coin RFID tags. a) Tags distant from each other $(>10 \mathrm{~cm})$. b) Medium distance tags $(5-10 \mathrm{~cm})$. c) Short distance tags $(<5 \mathrm{~cm})$. 
tags. When testing with the biocompatible crystal tags the quality of the received signal varied depending on the dimensions of the tags. While the $22 \mathrm{~mm}$ tags were easily detected and properly positioned by the system, it was not possible to detect the $12 \mathrm{~mm}$ ones in most cases.

The discrepancies in signal strength among different tags is due the different induction coefficient their internal antennas have. A close matching between the reader antenna and the tags' antennas is needed in order to achieve maximum signal transmission. This will be solved in future revisions by redesigning the geometry of the antennas with a smaller diameter and higher turn count.

\section{CONCLUSIONS}

In order to complement current visual tracking systems, a radio frequency tracking system, which overcomes their limitations, was developed. The challenge was to create a RFID tracking system which could be combined with existing visual tracking systems in order to improve the location and identification of laboratory animals in controlled environments.

The RATT system was designed to take into account the difficulties of experimenting with movement of small animals. This system is composed of electronic tiles, which have RFID antennas, to improve the tracking and the identification of the animals. It was designed in a modular fashion in order to be adaptable and easily maintained.

The RATT system, as well as providing identification functions, gives coverage in places where visual tracking is not possible. This vastly improves the quality of the results of behavior research studies.

\section{REFERENCES}

[1] T. Wey, D. T. Blumstein, W. Shen, and F. Jordán, "Social network analysis of animal behaviour: a promising tool for the study of sociality,” Anim. Behav., vol. 75, no. 2, pp. 333-344, 2008.

[2] R. J. Bodnar, "Biological Psychology: An Introduction to Behavioral, Cognitive, and Clinical Neuroscience," JAMA, vol. 298, no. 22, pp. 2680-2686, 2007.

[3] L. Lewejohann, A. M. Hoppmann, P. Kegel, M. Kritzler, A. Krüger, and N. Sachser, "Behavioral phenotyping of a murine model of Alzheimer's disease in a seminaturalistic environment using RFID tracking.,” Behav. Res. Methods, vol. 41, no. 3, pp. 850-856, 2009.

[4] R. C. Hinz and G. G. de Polavieja, "Ontogeny of collective behavior reveals a simple attraction rule,” Proc. Natl. Acad. Sci. , Feb. 2017.

[5] A. T. Schaefer and A. Claridge-Chang, "The surveillance state of behavioral automation.," Curr. Opin. Neurobiol., vol. 22, no. 1, pp. 170-6, Feb. 2012.

[6] E. Dreosti, G. Lopes, A. R. Kampff, and S. W. Wilson, "Development of social behaviour in young zebrafish.," Front Neural Circuits, vol. 9, no. Aug., p. 39, 2015.

[7] U. M. Jow, P. McMenamin, M. Kiani, J. R. Manns, and M. Ghovanloo, "EnerCage: A smart experimental arena with scalable architecture for behavioral experiments," IEEE Trans. Biomed. Eng., vol. 61, no. 1, pp. 139-148, 2014.

[8] A. Pérez-Escudero, J. Vicente-Page, R. C. Hinz, S. Arganda, and G. G. de Polavieja, "idTracker: tracking individuals in a group by automatic identification of unmarked animals.," Nat. Methods, vol. 11, no. 7, pp. 743-8, 2014.

[9] S. Ohayon, O. Avni, A. L. Taylor, P. Perona, and S. E. Roian Egnor, "Automated multi-day tracking of marked mice for the analysis of social behaviour,” J. Neurosci. Methods, vol. 219, no. 1, pp. 10-19, 2013.
[10] A. Weissbrod et al., "Automated long-term tracking and social behavioural phenotyping of animal colonies within a semi-natural environment.," Nat. Commun., vol. 4, no. May, p. 2018, 2013.

[11] A. Isasi, S. Rodriguez, J. L. D. Armentia, and A. Villodas, "Location, tracking and identification with RFID and vision data fusion," European Workshop on Smart Objects: Systems, Technologies and Applications. pp. 1-6, 2010.

[12] Y. Sztainberg and A. Chen, "An environmental enrichment model for mice.,” Nat. Protoc., vol. 5, no. 9, pp. 1535-1539, 2010.

[13] R. Tesoriero, J. A. Gallud, M. D. Lozano, and V. M. R. Penichet, “Tracking autonomous entities using RFID technology," IEEE Trans. Consum. Electron., vol. 55, no. 2, 2009.

[14] A. W. Reza, T. K. Geok, and K. Dimyati, "Tracking via Square Grid of RFID Reader Positioning and Diffusion Algorithm,” Wirel. Pers. Commun., vol. 61, no. 1, pp. 227-250, 2011. 